\title{
Vegetation Map of South Africa, Lesotho and Swaziland 2009 and 2012: A description of changes from 2006
}

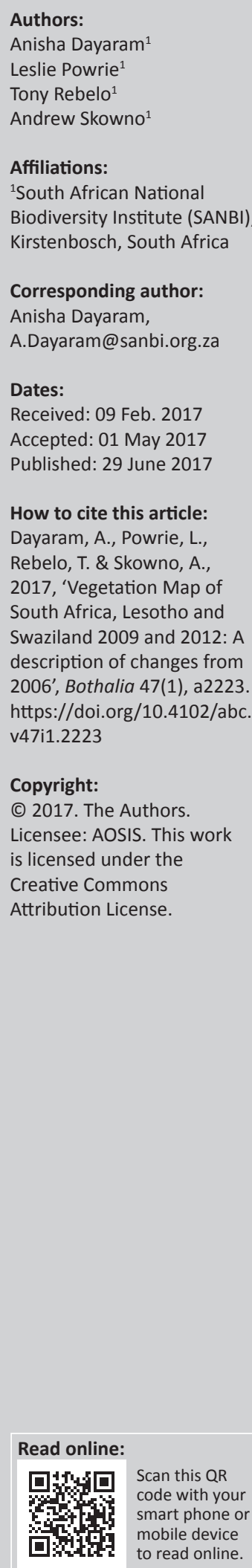

Background: The variety of applications in which the Vegetation Map of South Africa, Lesotho and Swaziland (VEGMAP) is used requires the map to be continually updated and refined to reflect the latest available information. The VEGMAP has been updated twice, in 2009 and 2012, since its first release in 2006.

Objectives: The first objective is to report on the motivations for changes in the 2009 and 2012 versions. The second objective is to describe new vegetation types and subtypes included in these versions.

Method: Changes to the VEGMAP are implemented after a peer-review process that is managed by the National Vegetation Map Committee. Accepted changes are then incorporated into the VEGMAP using GIS software.

Results: Seventy-one of the 449 vegetation types were affected by updates. Changes included the addition of new vegetation types and subtypes, modifications to the boundaries of types present in the 2006 VEGMAP and changes to the names of vegetation types.

Conclusion: The updates have affected a small portion of the map but have reflected a progressive refinement in quality. Regions that are still mapped at a coarse scale, especially those earmarked for land-use development, should be prioritised for improved map accuracy and classification through a more proactive approach towards vegetation mapping, using guidelines that are under development.

\section{Introduction}

The National Vegetation Map of South Africa, Lesotho and Swaziland (VEGMAP) (ed. Mucina \& Rutherford 2006) is a geographical classification of groups of plant communities across South Africa. The project was a collaboration between vegetation experts in the early 2000s under the custodianship of the South African National Biodiversity Institute (SANBI). Uses for the VEGMAP are widespread and cross-sectorial. Therefore, SANBI has a responsibility to ensure that the map reflects the most up-to-date knowledge available. Similar to early versions of vegetation maps in other parts of the world (Barbour, Todd Keeler-Wolf \& Schoenherr 2007), the first version of the VEGMAP was based on the best available data at the time and implemented through a largely top-down approach as vast regions of the country had been poorly sampled. While some areas of the map were developed from existing fine scale maps and floristic data $(\sim 6 \%$ of the map), much of the vegetation map was inferred from environmental predictors of floristic biogeography such as geology $(\sim 8 \%)$, climate $(\sim 5 \%)$, topography $(\sim 0.1 \%)$, bioresource units $(\sim 5 \%)$, land types $(\sim 42 \%)$ and satellite imagery $(\sim 7 \%)$ (Mucina, Rutherford \& Powrie 2006). Consequently, the VEGMAP is effective in representing natural vegetation communities at the national scale, but the top-down classification is less effective at the local scale (Barbour et al. 2007; Greenberg et al. 2006). There is thus a process through which the National Vegetation Map can be updated to improve its mapping accuracy in large parts of the country.

The 2009 and 2012 updates of the VEGMAP drew on suggestions and queries submitted by botanists and ecologists working at SANBI and various partner organisations. These versions were made available on the Biodiversity GIS (BGIS) website (www.bgis.org.za) since 2009, where full descriptions of each new vegetation type were recently (2016) added as downloadable portable documents (pdf). However, the justifications for re-classification of vegetation types and additions of new types have not been formally published. This paper highlights areas edited in the 2009 and the 2012 updates. We justify these changes and provide summary descriptions of the new vegetation types and subtypes. 


\section{Methods}

\section{Data requirements for changes to the Vegetation Map of South Africa, Lesotho and Swaziland}

Proposed changes require review and validation before they can be accepted. Therefore, a National Vegetation Map Committee (NVMC) was formed in 2007. Similar to other international classification committees, such as the vegetation subcommittee of the American Federal Geographic Data Committee (FGDC 2008) and the Canadian National Vegetation Classification Technical Committee, the NVMC provides technical direction for the VEGMAP project and ratifies proposed changes where sufficient justification has been submitted. The minimum information required for a proposed change will depend on the nature of the change. Minor changes include changes to the boundaries of existing vegetation types and updates to the descriptions. Major changes include the proposal of new vegetation types or merging of existing types. The minimum information required for a change to be accepted includes: (1) a digital map of proposed boundaries; (2) detailed justification for the various changes (often with satellite imagery, aerial photographs or orthophotographs); (3) for new vegetation types and subtypes the justification must include a broad description of the landscape, vegetation structure, associated geology, soils, landscape features and climate, lists of dominant and diagnostic species, conservation status and (optionally) threats; and (4) a georeferenced colour photograph with a caption. Major changes must be accompanied by a peer reviewed publication or report.

\section{Results}

\section{Summary of changes to the Vegetation Map of South Africa, Lesotho and Swaziland}

The sources of changes implemented in the updates include vegetation mapping for fine scale planning (Helme 2007a, 2007b, 2007c; Helme \& Koopman 2007; Van der Merwe et al. 2008a, 2008b; Vlok, Cowling \& Wolf 2005), provincial planning (Scott-Shaw \& Escott 2011) and field work conducted by members of the NVMC. Accepted changes to the VEGMAP have included alterations to the boundaries of vegetation types classified in 2006: changes to the spelling of vegetation type names (4), the addition of new vegetation types (11) and the addition of subtypes of existing vegetation types (9). The reasons for changes ranged from ground truthed (direct observation of vegetation patterns in the field) evidence to evidence based on re-analysis using updated satellite imagery (Table 1).

Ground-truthing was the most common form of evidence (90\% of the affected vegetation types) used to justify changes, often supplemented by information on floristic, geomorphological, structural or geological distinctness. Minor changes in 2012 included changing the names of four vegetation types to remove the circumflex $(\wedge)$ from the region name 'Rûens'. This was aimed at eliminating errors in computer syntax.
The boundaries of 43 vegetation types were edited in the 2009 version and four in the 2012 version. New vegetation types were added mainly in KwaZulu-Natal, Mpumalanga and the Western Cape provinces (Figure 1). Six new vegetation types and nine subtypes (mainly in the Western and Northern Cape provinces) were added in 2009, and six types were added in 2012. Four of the new vegetation types (SKk9, SV125, SV126 and SV127) were described in the Succulent Karoo biome, with two each from the Fynbos (FFs32, FFh11) and Grassland (Gs19, Gs20) biomes, and one from the Indian Ocean Coastal Belt (CB6). One vegetation type (FFa5 Olifants Alluvium Fynbos) was added in 2009 from fine scale planning, but it was removed in 2012 as supporting evidence was insufficient to justify the change. Three of the five sources of accepted updates emerged out of provincial or city-funded fine scale planning projects. New polygons were added (W5 Reclaimed Land) to represent the current extent of a small portion of coast in Table Bay that was filled in during upgrades to Cape Town Harbour in the 1940s. This area was not historically vegetated and is mapped but not considered to be a vegetation type.

A condensed version of the descriptions for new vegetation types and subtypes added in 2009 and 2012 versions is outlined in Tables 2 and 3.

\section{Discussion}

Overall, the 2009 and 2012 versions of the VEGMAP have been progressive refinements in scale and accuracy compared to the 2006 version, although these are limited to a few regions. Recent versions have also allowed for the inclusion of finer scale data in the form of subtypes. A problem remains as when to allow subtypes in a way that prevents a proliferation of vegetation units that will compromise the national scale of VEGMAP. Subtypes vary in scale, and in the criteria used to define them; thus some subtypes are represented by several units spread over larger geographies (e.g. FS1.2 Graafwater Flats Strandveld), while others are represented by a single isolated unit (e.g. FRs8.2 Kluitjieskraal Silcrete Renosterveld). This has the potential to become problematic as the units for types are refined, and approach the same scale as the units currently mapped as subtypes. This occurrence can only be avoided by a clear and welldefined classification hierarchy.

The VEGMAP changes to date were driven by a relatively passive approach to data acquisition, from private botanical consultants and government conservation officials raising concerns about the current map, and providing information from fine scale planning work. Consequently, updates have been concentrated in areas where funding, capacity and historical baseline information was available. Areas that have been updated so far coincided with three principal regions and two centres of plant endemism, namely, Kamiesberg Centre of the Cape Floristic Region, the Little Karoo Centre of the Succulent Karoo Region and a large extent of the Maputaland-Pondoland Region (Steenkamp et al. 2005). 
TABLE 1: The South African vegetation types updated in 2009 and 2012 grouped by change category. Subscripts are referenced below the table. Vegetation type (and code)

Nature of the update ('Other' includes: Geomorphologically distincta; disjunct community'; altitude, topographic position and climatic factors'; and ASCll character set transfer error

\begin{tabular}{cccccc}
\cline { 2 - 4 } & $\begin{array}{c}\text { Ground } \\
\text { truthed }\end{array}$ & $\begin{array}{c}\text { Floristically } \\
\text { distinct }\end{array}$ & $\begin{array}{c}\text { Geologically } \\
\text { distinct }\end{array}$ & $\begin{array}{c}\text { Structurally } \\
\text { distinct }\end{array}$ & $\begin{array}{c}\text { Supported by } \\
\text { Satellite imagery }\end{array}$ \\
\hline
\end{tabular}

\section{Polygon boundary adjustment}

Agter-Sederberg Shrubland SKv ${ }^{1}$

Bokkeveld Sandstone Fynbos FFs1

Cape Estuarine Salt Marshes Aze2

Cape Inland Salt Pans AZi9

Cape Lowland Freshwater Wetland AZf $1^{1}$

Cape Seashore Vegetation AZd ${ }^{1}$

Cederberg Sandstone Fynbos FFs4 ${ }^{1}$

Cederberg Sandstone Fynbos FFs $4^{1}$

Citrusdal Vygieveld SKk7 $7^{1}$

Doringrivier Quartzite Karoo SKv1 ${ }^{1}$

Graafwater Sandstone Fynbos FFs ${ }^{1}$

Hantam Karoo SKt2

Hopefield Sand Fynbos FFd3 ${ }^{1}$

Klawer Sandy Shrubland SKs $13^{1}$

Knersvlakte Dolomite Vygieveld SKk6 ${ }^{1}$

Knersvlakte Quartz Vygieveld SKk3 ${ }^{1}$

Knersvlakte Shale Vygieveld SKk4

KwaZulu-Natal Sandstone Sourveld SVs $5^{4}$

Lamberts Bay Strandveld FS ${ }^{1}$

Langebaan Dune Strandveld FS5 ${ }^{1}$

Leipoldtville Sand Fynbos FFd2 ${ }^{1}$

Little Karoo Quartz Vygieveld SKv10 $10^{6}$

Midlands Mistbelt Grassland Gs9 ${ }^{4}$

Namaqualand Riviere AZi1

Namaqualand Sand Fynbos FFd $1^{1}$

Namaqualand Spinescent Grassland SKs12

Namaqualand Strandveld SKs $7^{1}$

Nieuwoudtville Shale Renosterveld FRs $2^{1}$

Northern Inland Shale Band Vegetation FFb1

Northern Zululand Mistbelt Grassland Gs1

Olifants Sandstone Fynbos FFs $3^{1}$

Paulpietersburg Moist Grassland Gm154

Piketberg Quartz Succulent Shrubland SKk8

Piketberg Sandstone Fynbos FFs6 ${ }^{1}$

Robertson Karoo SKv7

Roggeveld Shale Renosterveld FRs $3^{5}$

Saldanha Flats Strandveld FS3 ${ }^{2}$

Saldanha Granite Strandveld FS2 ${ }^{2}$

Saldanha Limestone Strandveld FS4 ${ }^{2}$

Southern Afrotemperate Forest FOz $1^{1}$

Southern Drakensberg Highland Grassland Gd4

Swartland Shale Renosterveld FRs9 ${ }^{1}$

Swartland Silcrete Renosterveld FRc1 ${ }^{1}$

Vanrhynsdorp Gannabosveld SKk $5^{1}$

Winterhoek Sandstone Fynbos FFs5 ${ }^{1}$

Zululand Lowveld SVL23 ${ }^{4}$

$+$

\section{New vegetation type}

Citrusdal Shale Renosterveld FRs $20^{1}$

Dry Coast Hinterland Grassland Gs $19^{4}$

Kobee Succulent Shrubland SKk9 ${ }^{1}$

KwaZulu-Natal Coastal Belt Thornveld CB6 ${ }^{4}$

Long Tom Pass Montane Grassland Gm31 ${ }^{4}$

Maputaland Pallid Sandy Bushveld SVI $25^{4}$

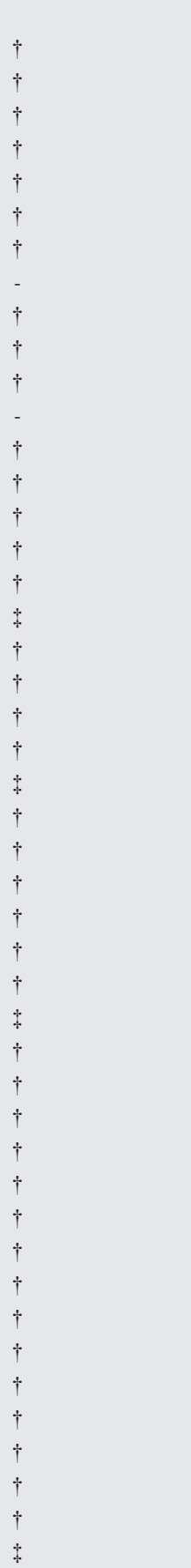

(n)

$\dagger+$

$+$

Source: ${ }^{1}$ Helme 2007a, 2007b, 2007c; ${ }^{2}$ Helme and Koopman 2007; ${ }^{3}$ NVMC decision; ${ }^{4}$ Scott-Shaw and Escott 2011; ${ }^{5}$ Van der Merwe et al. 2008a, 2008b; ${ }^{6}$ Vlok et al. 2005

a, Geomorphologically distinct;

b, disjunct community;

c, altitude, topographic position and climatic factors;

d, ASCII character set transfer error.

$\dagger$, updated in 2009; \$, updated in 2012; $\S$, added in 2009 and removed in 2012. 
TABLE 1 (Continues...): The South African vegetation types updated in 2009 and 2012 grouped by change category. Subscripts are referenced below the table. Vegetation type (and code) Nature of the update ('Other' includes: Geomorphologically distinct ${ }^{\mathrm{a}}$; disjunct community'; altitude, topographic position and climatic factors'; and ASCII character set transfer error ${ }^{d}$ )

\begin{tabular}{|c|c|c|c|c|c|c|}
\hline & \\
\hline & $\begin{array}{l}\text { Ground } \\
\text { truthed }\end{array}$ & $\begin{array}{l}\text { Floristically } \\
\text { distinct }\end{array}$ & $\begin{array}{l}\text { Geologically } \\
\text { distinct }\end{array}$ & $\begin{array}{c}\text { Structurally } \\
\text { distinct }\end{array}$ & $\begin{array}{c}\text { Supported by } \\
\text { Satellite imagery }\end{array}$ & Other \\
\hline Moist Coast Hinterland Grassland Gs2 ${ }^{4}$ & $\$$ & $\$$ & - & - & - & - \\
\hline Muzi Palm Veld and Wooded Grassland SVI26 & $\$$ & - & $\ddagger$ & - & $\ddagger$ & - \\
\hline Nardouw Sandstone Fynbos FFs $32^{1}$ & $\dagger$ & $\dagger$ & - & $\dagger$ & - & - \\
\hline Olifants Alluvium Fynbos FFa5 ${ }^{1}$ & $\S$ & - & - & - & - & - \\
\hline Peninsula Shale Fynbos FFh $11^{3}$ & $\$$ & $\$$ & - & $t$ & - & - \\
\hline Steenkampsberg Montane Grassland $\mathrm{Gm} 30^{4}$ & $\dagger$ & $\dagger$ & - & - & - & - \\
\hline \multicolumn{7}{|l|}{ Subtype of Vegetation type } \\
\hline Graafwater Flats Strandveld FS1.2 ${ }^{1}$ & $\dagger$ & $\dagger$ & - & - & - & - \\
\hline Hantam Escarpment Shrubland SKt2. $2^{5}$ & $\dagger$ & - & - & - & - & - \\
\hline Kluitjieskraal Silcrete Renosterveld FRs $8.2^{1}$ & $\dagger$ & $\dagger$ & - & - & - & - \\
\hline Nekkies Silty Floodplain FFa2. $2^{1}$ & $\dagger$ & $\dagger$ & - & - & - & - \\
\hline Nieuwoudtville Sandstone Renosterveld FRs $2.2^{1}$ & $\dagger$ & - & - & $\dagger$ & - & $\dagger^{a}$ \\
\hline Noord Bokkeveld Shale Renosterveld FRs $2.3^{1}$ & $\dagger$ & - & - & $\dagger$ & - & $\dagger^{a}$ \\
\hline Papkuilsfontein Sandstone Renosterveld FRs $2.4^{1}$ & $\dagger$ & - & - & $\dagger$ & - & $\dagger^{a}$ \\
\hline Varkvlei Shale Strandveld FS3. $2^{1}$ & $\dagger$ & - & $\dagger$ & - & - & - \\
\hline Stormsvlei Aalwyn Fynbos FFs $13.2^{1}$ & $\dagger$ & $\dagger$ & - & - & - & - \\
\hline \multicolumn{7}{|l|}{ Vegetation type name change } \\
\hline Central Ruens Shale Renosterveld FRs $12^{3}$ & - & - & - & - & - & $t^{d}$ \\
\hline Eastern Ruens Shale Renosterveld FRs $13^{3}$ & - & - & - & - & - & $t^{d}$ \\
\hline Ruens Silcrete Renosterveld FRc2 ${ }^{3}$ & - & - & - & - & - & $t^{d}$ \\
\hline Western Ruens Shale Renosterveld FRs $11^{3}$ & - & - & - & - & - & $t^{\mathrm{d}}$ \\
\hline
\end{tabular}

Source: ${ }^{1}$ Helme 2007a, 2007b, 2007c; ${ }^{2}$ Helme and Koopman 2007; ${ }^{3} \mathrm{NVMC}$ decision; ${ }^{4}$ Scott-Shaw and Escott 2011; ${ }^{5}$ Van der Merwe et al. $2008 \mathrm{a}$, $2008 \mathrm{~b} ;{ }^{6} \mathrm{Vlok}$ et al. 2005

a, Geomorphologically distinct;

b, disjunct community;

, altitude, topographic position and climatic factors:

d, ASCll character set transfer error.

$\dagger$, updated in 2009; + , updated in 2012; §, added in 2009 and removed in 2012

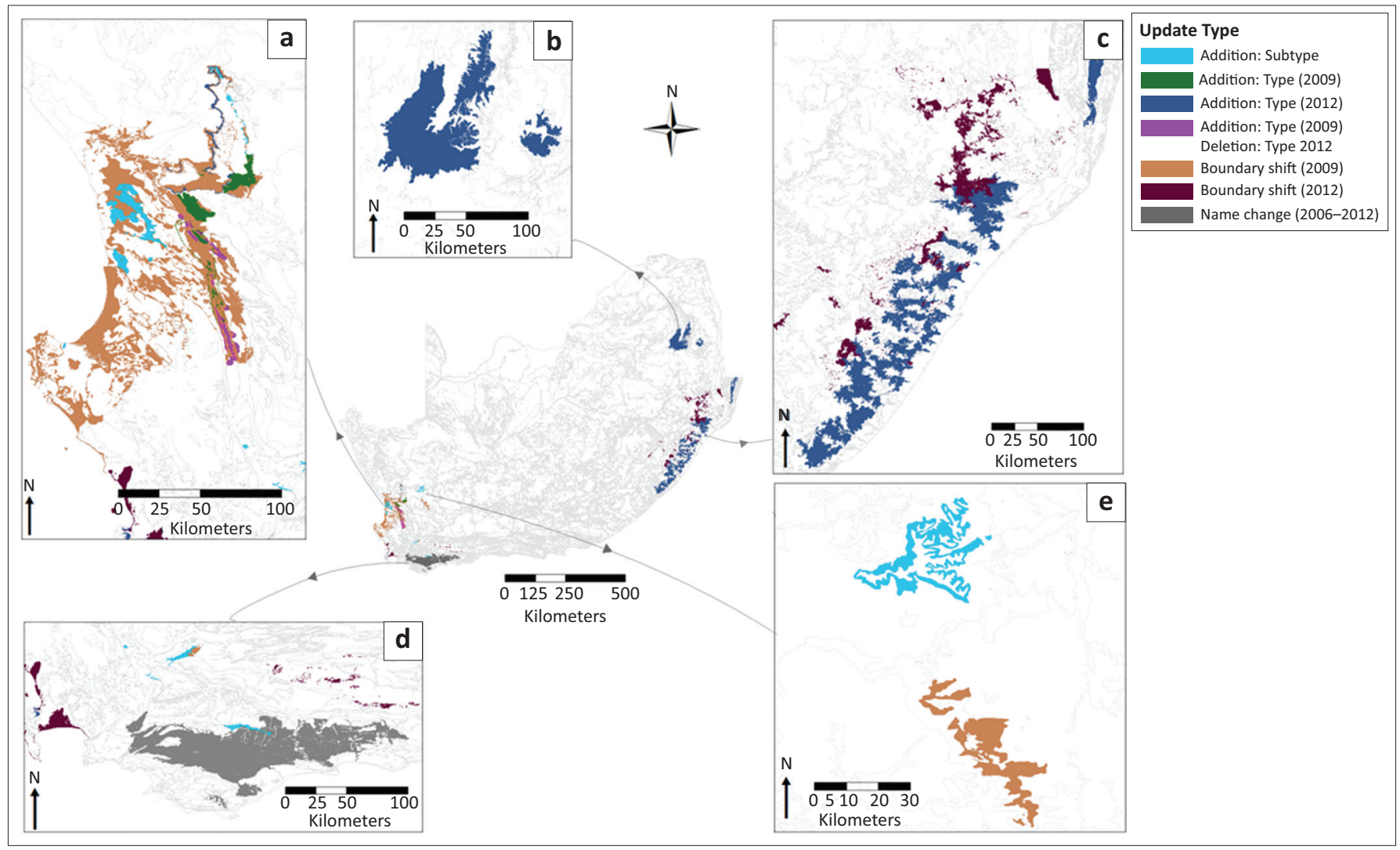

Source: These changes were based on evidence from the following sources: (a) Helme (2007), (b) ground truthed evidence from members of the National Vegetation Map Committee, (c) Scott-Shaw and Escott (2011), (d) Vlok et al. (2005) and (e) Van der Merwe et al. (2008a, 2008b)

FIGURE 1: The edited areas of the South African VEGMAP affected by the 2009 and 2012 updates. 


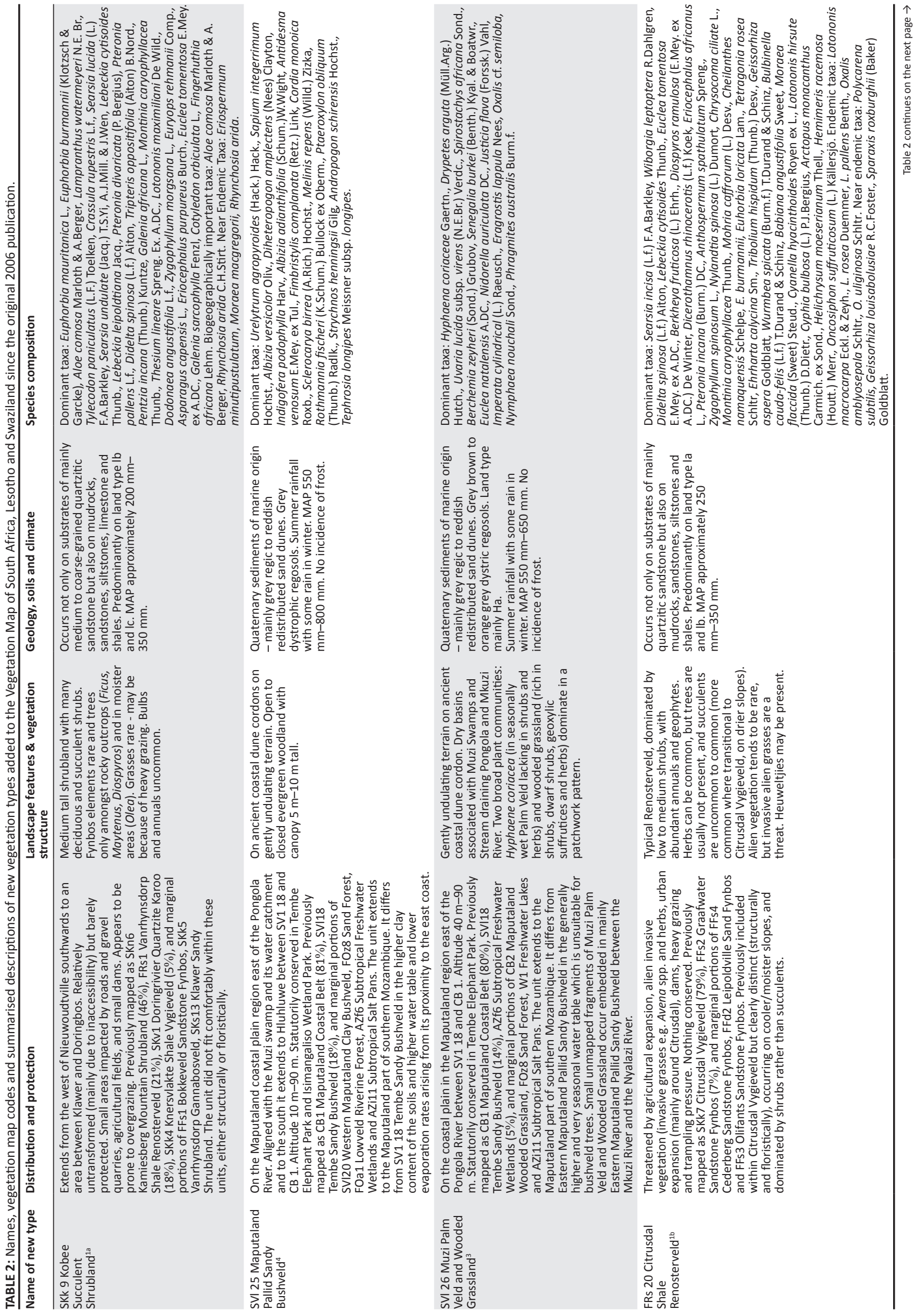




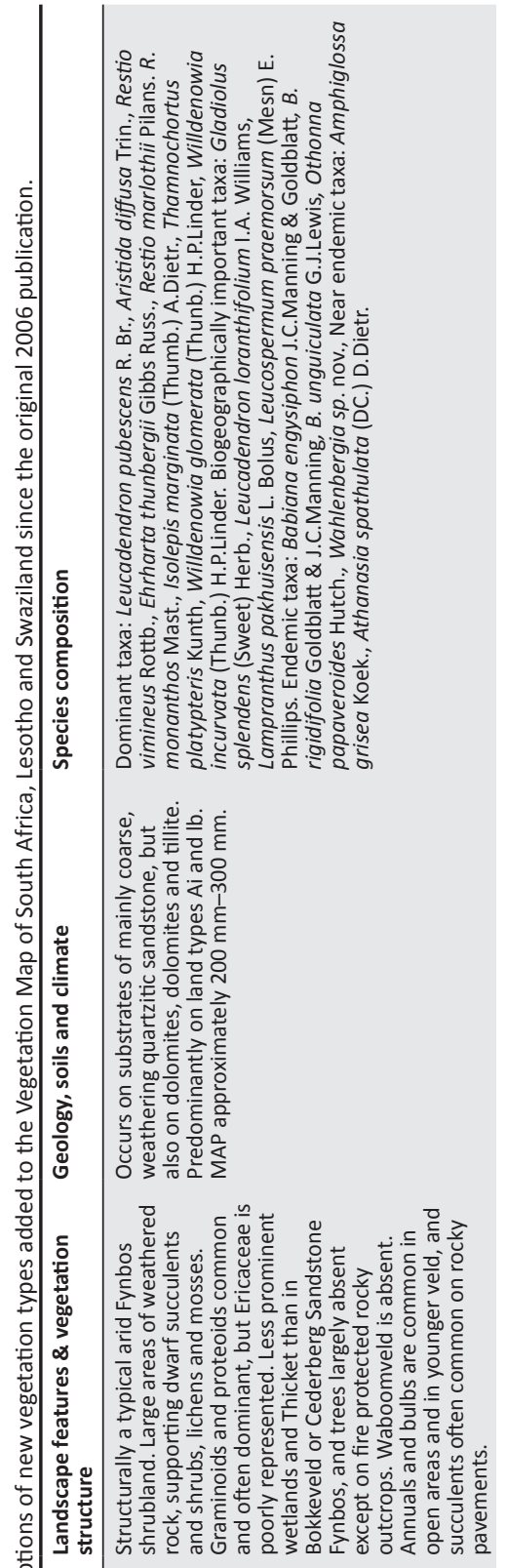

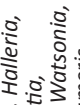

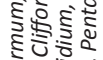

จ.

ड़े

के ำำ

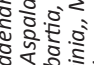

施.

o

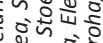

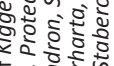

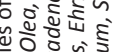

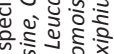

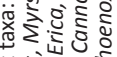

นิำ

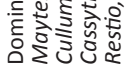

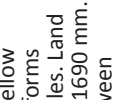

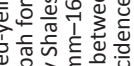

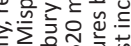

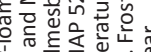

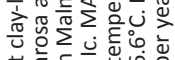

茄语

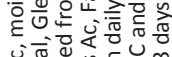

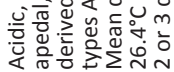

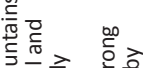

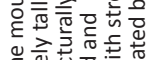

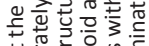

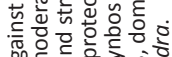

要施

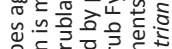

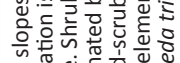

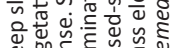

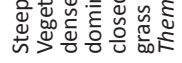

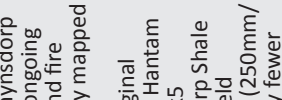

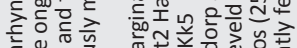

다에

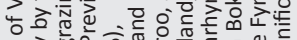

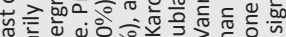

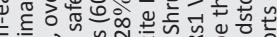

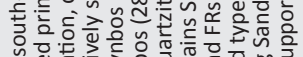

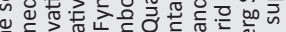

呵

든

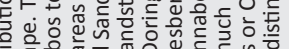

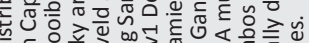

ֻ

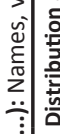

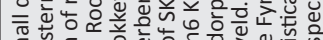

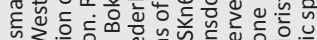

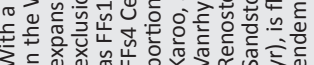

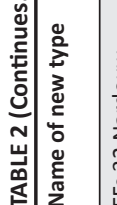

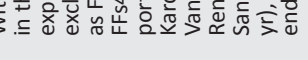

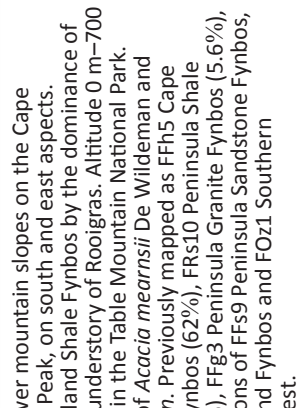

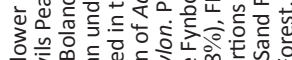

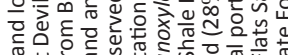

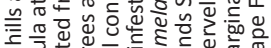

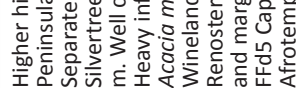

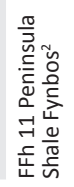

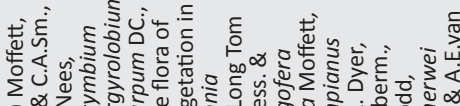

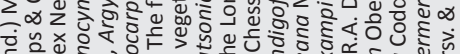

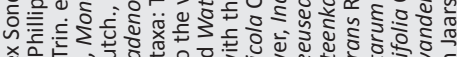

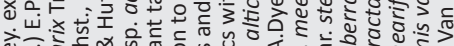

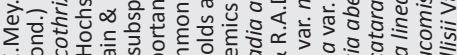

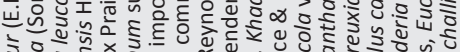

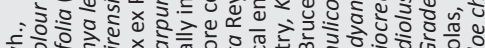

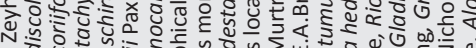
$\infty$

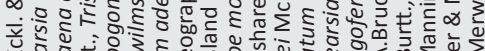

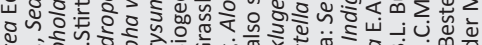

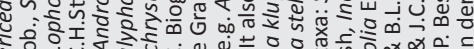

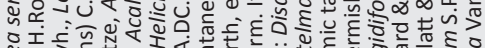

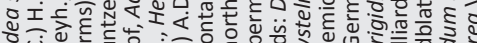

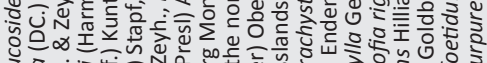

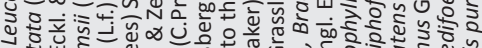

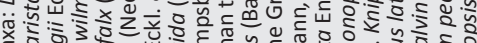
西: कि

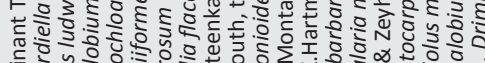

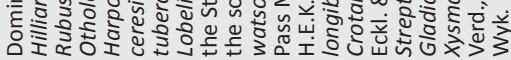

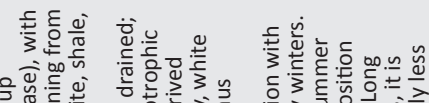

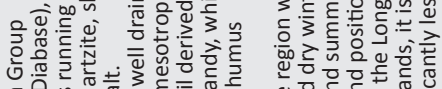

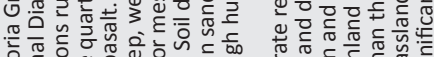

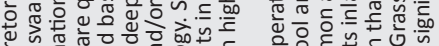

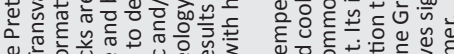

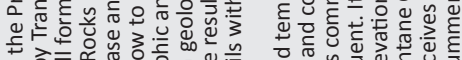

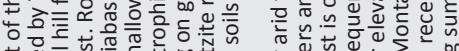

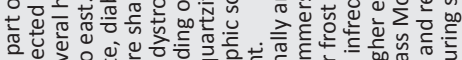

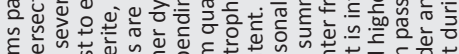

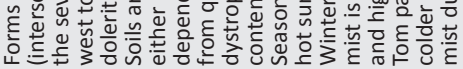

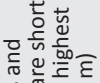

에에

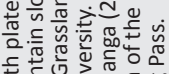

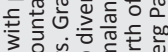

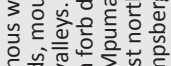

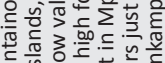

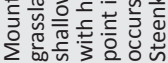

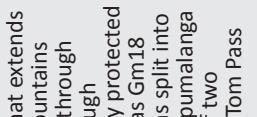

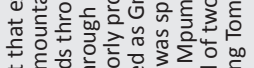

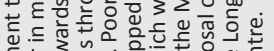

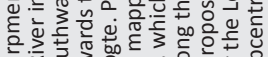

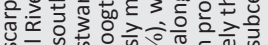

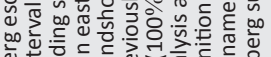

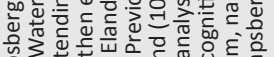

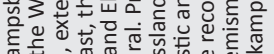

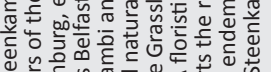

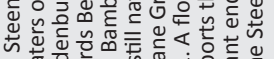

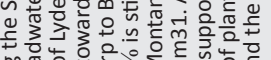

年

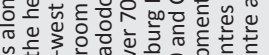

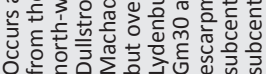

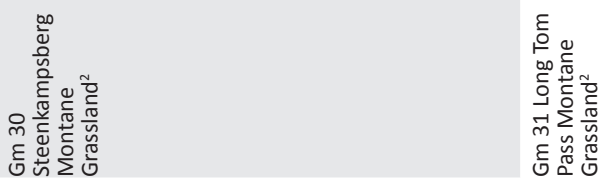

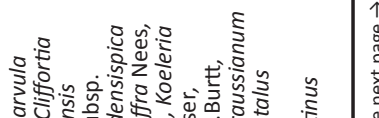

20.5

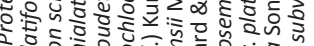

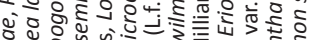

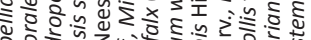

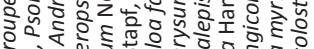

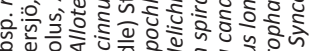

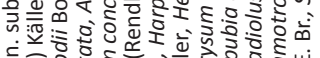

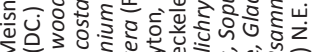

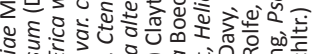

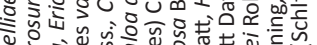

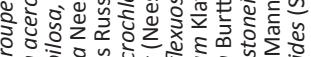

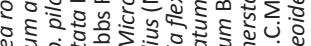

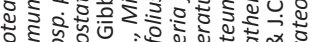

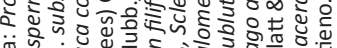

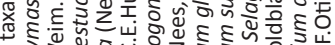

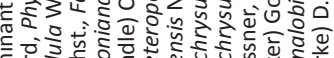

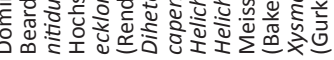

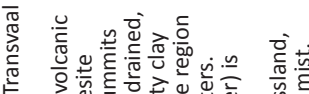

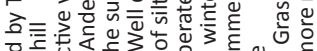

尊

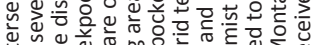

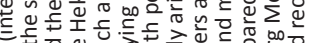

o

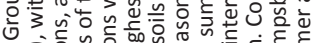
뜬.

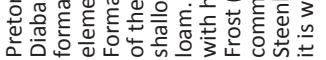

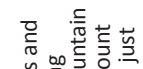

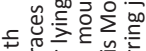

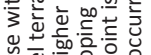

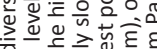

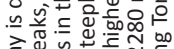

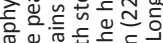

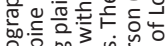

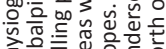

(1)

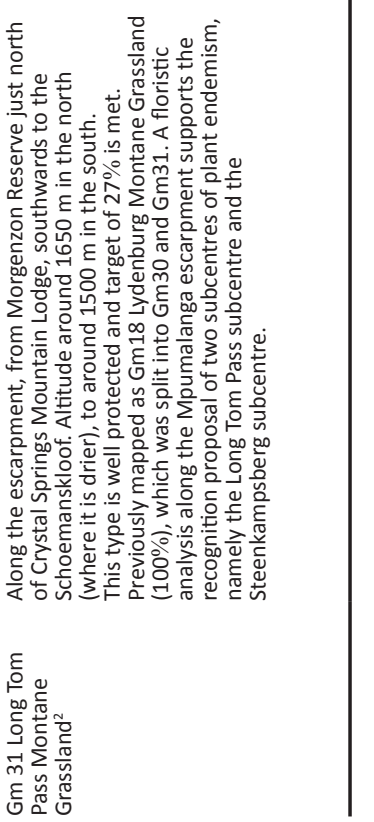




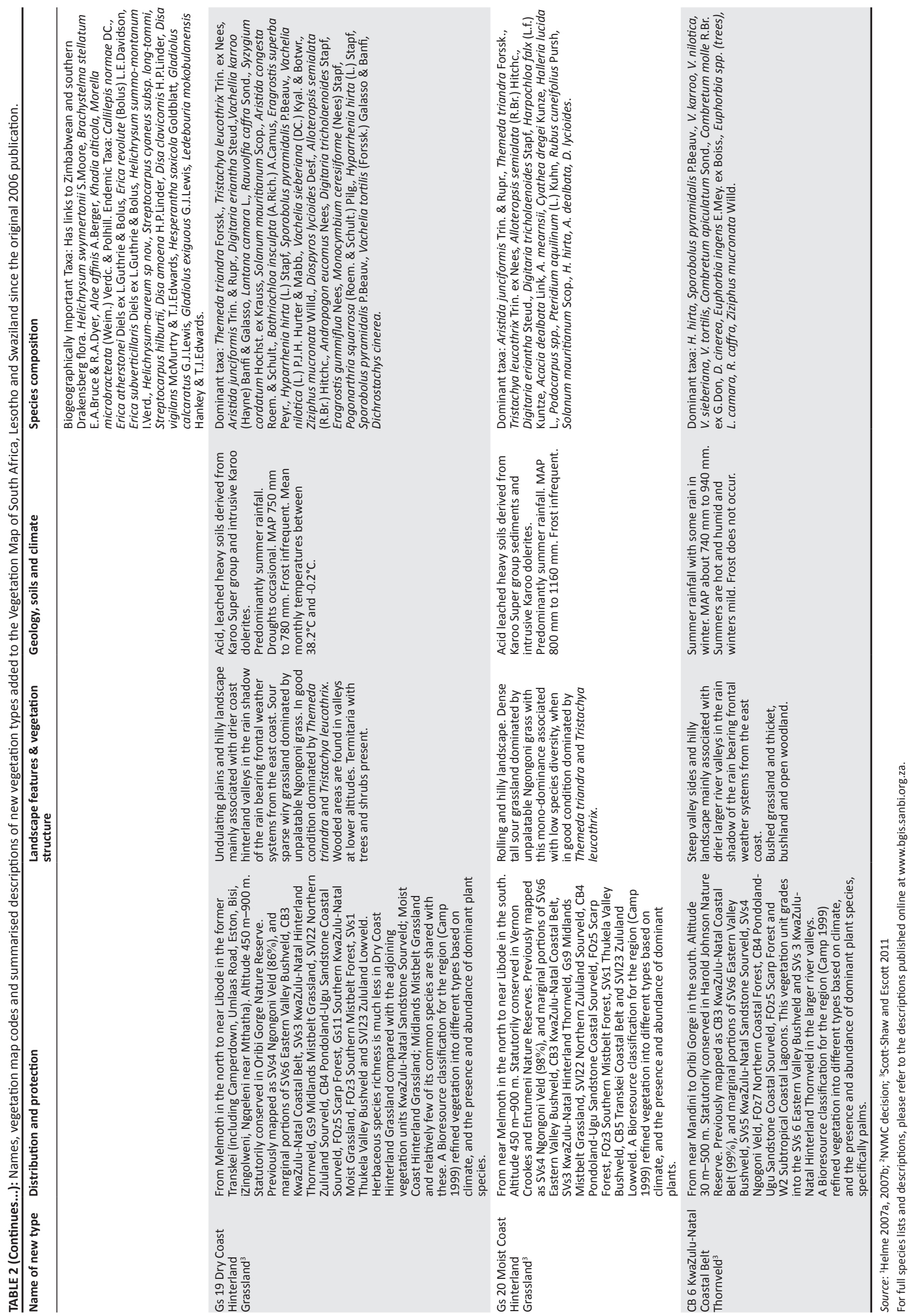




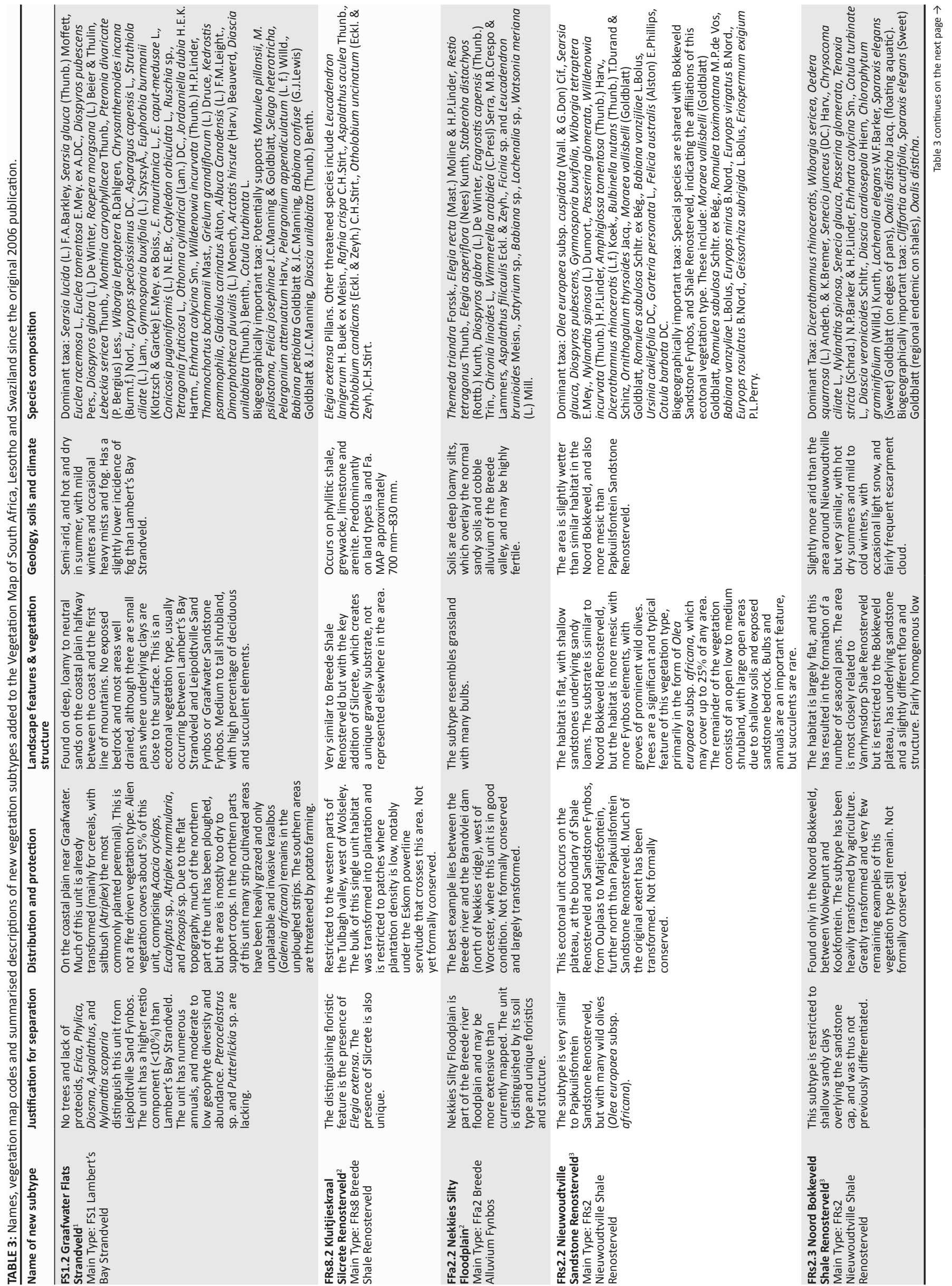




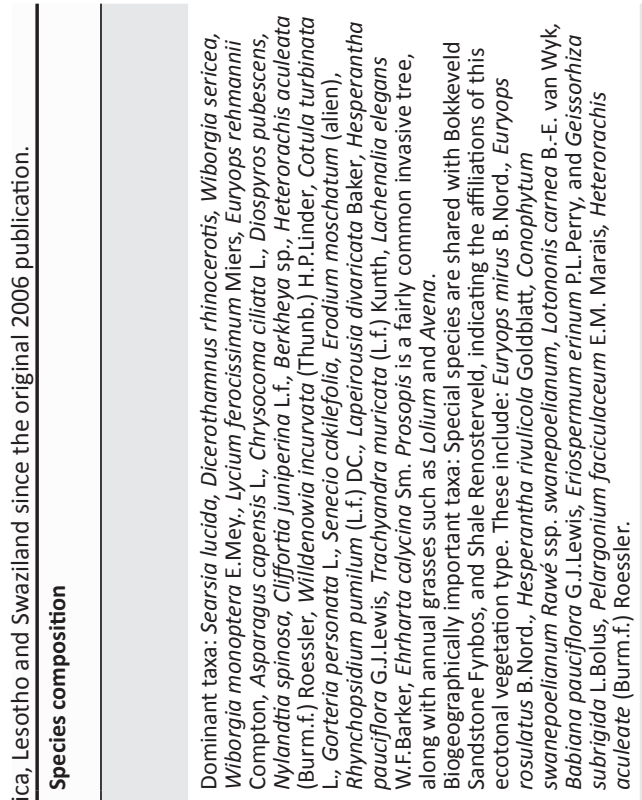

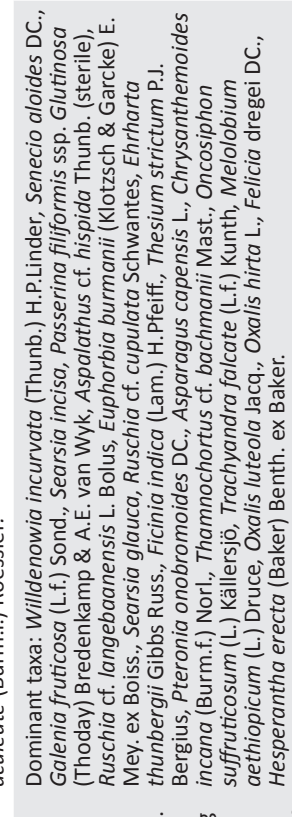

들

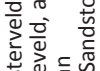

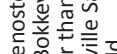

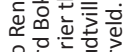

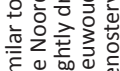

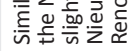

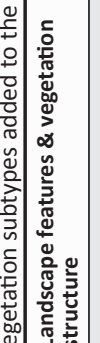

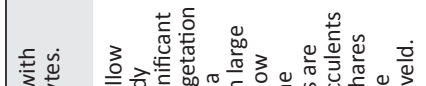

3)

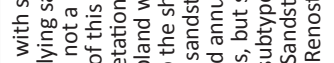

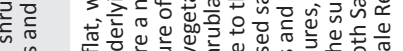

等

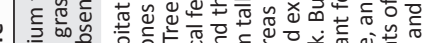

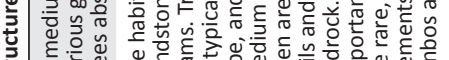

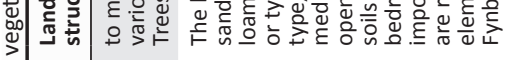

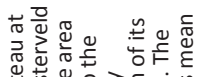

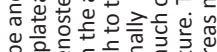

굴은.

家设

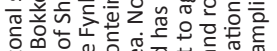

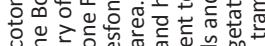

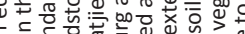

西

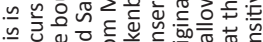

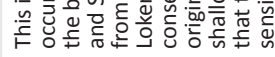

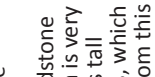

突

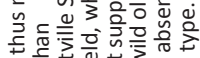

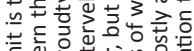

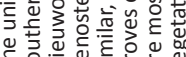

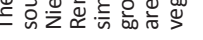

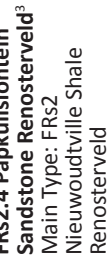

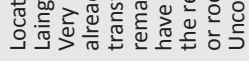

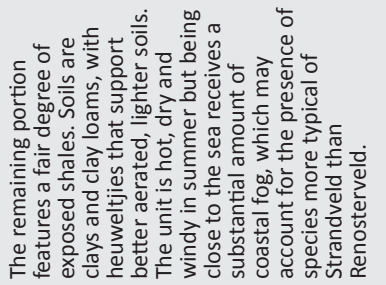

$\overline{\bar{\Sigma}}$

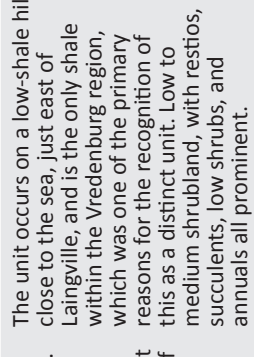

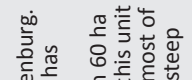

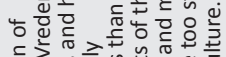

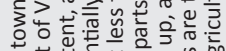

等

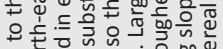

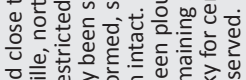

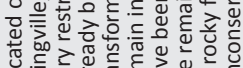

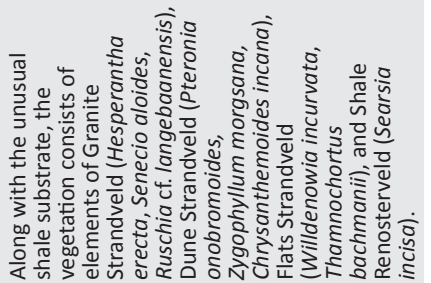

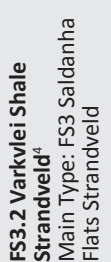

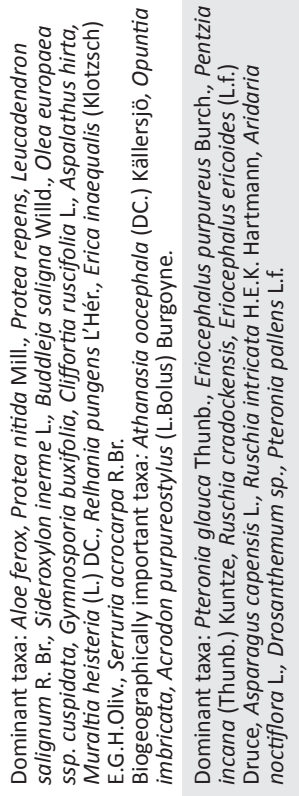

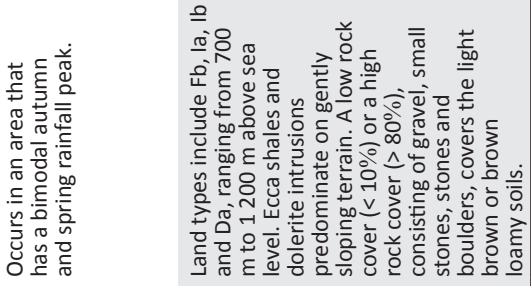

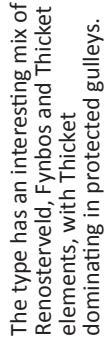

采

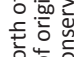

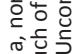

过 帘

हैं

次言

言产产

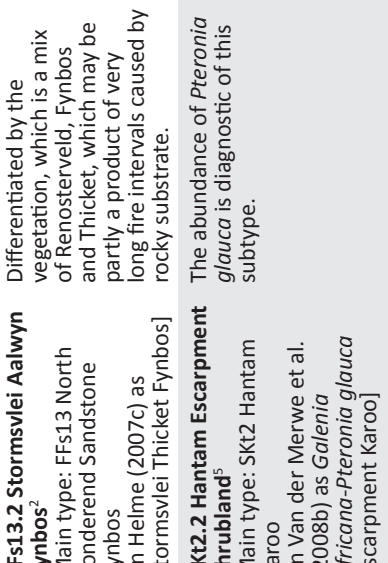

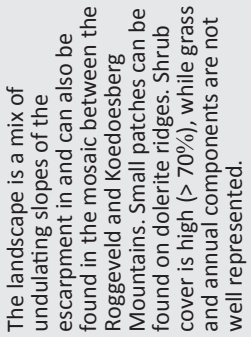

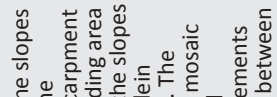

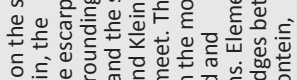

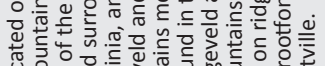

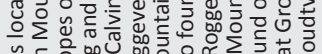
喵

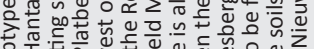

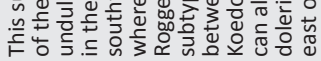

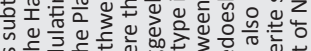


Twelve of the centres of plant endemism have not been refined in the nine-year update period. Of these, seven (i.e. Albany Centre, Drakensberg-alpine Centre, Barberton Centre, Wolkberg Center, Sekhukhuneland Centre, Soutpansberg Centre and Griqualand Centre) occur in the northern and central interiors of the country. These centres coincide with areas that either historically fell within the homeland territory (Wessels et al. 2004), are agricultural areas or are areas of economic development. Thus, botanical exploration has not been conducted to the same extent as in the eastern and western coastal regions where the updates have occurred. These unrefined parts of the northern, central interior and parts of the coastal Western Cape need to be prioritised for future refinement as the units in these regions are often large and were initially mapped at a coarse scale by inferring vegetation types from environmental patterns, e.g. land types were used to map many vegetation types in the Nama Karoo. In addition, many of these areas are in various stages of land-use transformation caused by urban and industrial development (Donaldson 2006), platinum and coal mining (Jeffrey 2005; Armitage, McDonald \& Tredoux 2007), the installation of solar and wind farms (Sparks et al. 2014) and shale gas development activities (Greef 2012).

To improve the VEGMAP in these regions, we need to streamline a process of data collection from the mapping of units to the submission and acceptance of updates, while providing reasonable standards to maintain data quality. To this end, we are developing a defined classification hierarchy for the VEGMAP, explicit standards to guide the mapping of VEGMAP units across each of the biomes and a set of guidelines for submitting and accepting updates to the VEGMAP. We are also exploring more proactive approaches to narrowing gaps between the expert driven classification that underpins the current VEGMAP and the actual communities that they represent.

\section{Acknowledgements}

The Vegetation Map Project is a large collaborative project that has spanned over two decades. Many have contributed to the success of this project and are too numerous to name individually; however, they are acknowledged in the original publication. The authors wish to thank Mr Nick Helme, Dr Helga van der Merwe, the late Mr Robb Scott-Shaw, Mr Boyd Escott and Mr Jan Vlok for sharing data that contributed to the updated areas in the 2009 and 2012 versions of the vegetation map. The authors also thank Emily Botts for proofreading.

\section{Competing interests}

The authors declare that they have no financial or personal relationships that may have inappropriately influenced them in writing this article.

\section{Authors' contributions}

A.D. prepared the draft; L.P. implemented the bulk of the technical changes to the map; and T.R. and A.S. edited the text.

\section{References}

Armitage, P., McDonald, I. \& Tredoux, M., 2007, 'A geological investigation of the waterberg hydrothermal platinum deposit, Mookgophong, Limpopo Province, South Africa', Applied Earth Science 116(3), 113-129. https://doi.org/10.1179/ $174327507 \times 207483$

Barbour, M.G., Todd Keeler-Wolf, T. \& Schoenherr, A.A. (eds.), 2007, Terrestrial vegetation of California, University of California Press, London, England.

Camp, K.G.T., 1999, 'A bioresource classification for KwaZulu-Natal, South Africa', MSc thesis, School of Applied Environmental Resources, University of Natal.

Donaldson, R., 2006, 'Mass rapid rail development in South Africa's metropolitan core: Towards a new urban form?', Land Use Policy 23, 344-352. https://doi. org/10.1016/j.landusepol.2005.02.003

Federal Geographic Data Committee (FGDC), 2008, National vegetation classification standard (version 2.0): FGDC document number FGDC-STD-005-2008, US Geological Survey, Reston, VA.

Greef, L., 2012, You can't have your gas and drink your water! Hydraulic fracturing in the context of South Africa's looming water crisis, EMG Water and Climate Change Research Series: Report 6, Environmental Monitoring Group, Observatory, Cape Town, South Africa.

Greenberg, J.A., Dobrowski, S.Z., Ramirez, C.M., Tuil, J.L. \& Ustin, S.L., 2006, 'A bottomup approach to vegetation mapping of the Lake Tahoe Basin using hyperspatial image analysis', Photogrammetric Engineering \& Remote Sensing 72(5), 581-589. https://doi.org/10.14358/PERS.72.5.581

Helme, N.A., 2007a, Botanical report: Fine scale vegetation mapping of the Bokkeveld Escarpment, C.A.P.E. Programme, CapeNature, Newlands, Cape Town.

Helme, N.A., 2007b, Botanical report: Fine scale vegetation mapping in the Sandveld, C.A.P.E. Programme, CapeNature, Newlands, Cape Town.

Helme, N.A., 2007c, Botanical report: Fine scale vegetation mapping in the Upper Breede River Valley, C.A.P.E. Programme, CapeNature, Newlands, Cape Town.

Helme, N.A. \& Koopman, R., 2007, Botanical report: Fine scale vegetation mapping in the Saldanha Peninsula, C.A.P.E. Programme, CapeNature, Newlands, Cape Town.

Jeffrey, L., 2005, 'Challenges associated with further development of the Waterberg Coalfield', The Journal of The South African Institute of Mining and Metallurgy 105, 453-458.

Mucina, L. \& Rutherford, M.C. (eds.), 2006, The vegetation of South Africa, Lesotho and Swaziland, South African National Biodiversity Institute, Pretoria.

Mucina, L., Rutherford, M.C. \& Powrie, L.W., 2006, 'Logic of the map: Approaches and procedures', in L. Mucina \& M.C. Rutherford (eds.), The vegetation of South Africa, Lesotho and Swaziland, pp. 12-29. SANBI, Pretoria.

Scott-Shaw, W.R. \& Escott, B.J. (eds.), 2011, 'KwaZulu-Natal provincial pretransformation vegetation type map - 2011', unpublished GIS Coverage [kznveg05v2 011 wll.zip], Biodiversity Conservation Planning Division, Ezemvelo KZN Wildlife, Cascades, Pietermaritzburg.

Sparks, D., Madhlopa, A., Keen, S., Moorlach, M., Dane, A., Krog, P. et al., 2014 'Renewable energy choices and their water requirements in South Africa', Journal of Energy in Southern Africa 25(4), 80-92.

Steenkamp, Y., Van Wyk, A.E., Smith, G.F. \& Steyn, H., 2005, 'Floral endemism in southern Africa: A numerical classification at generic level', in I. Friis \& H. Balslev (eds.), Plant diversity and complexity patterns: Local, regional and global dimensions: Proceedings of an international Symposium held at the Royal Danish Academy of Sciences and Letter, Copenhagen, Denmark, May 25-28, 2003, pp. 253-271.

Van der Merwe, H., Van Rooyen, M.W. \& Van Rooyen, N., 2008a, 'Vegetation of the Hantam-Tanqua-Roggeveld subregion, South Africa, Part 1: Fynbos Biome related vegetation', Koedoe 50, 61-71. https://doi.org/10.4102/koedoe.v50i1.130

Van der Merwe, H., Van Rooyen, M.W. \& Van Rooyen, N., 2008b, 'Vegetation of the Hantam-Tanqua-Roggeveld subregion, South Africa, Part 2: Succulent Karoo Biome related vegetation', Koedoe 50, 160-183. https://doi.org/10.4102/koedoe. v50i1.148

Vlok, J.H.J., Cowling, R.M. \& Wolf, T., 2005, A vegetation map for the Little Karoo, unpublished maps and report for a SKEP project supported by CEPF grant no 1064410304, Cape Town.

Wessels, K.J., Prince, S.D., Frost, P.E. \& Van Zyl, D., 2004, 'Assessing the effects of human-induced land degradation in the former homelands of northern South Africa with a 1 km AVHRR NDVI time-series', Remote Sensing of Environment 91 47-67. https://doi.org/10.1016/j.rse.2004.02.005 